\title{
ANALYTIC MULTIPLIERS OF THE RADON TRANSFORM
}

\author{
JAMES V. PETERS
}

\begin{abstract}
We define the Radon transform over the real and complex domain, and list some of its simplest properties. For the complex domain $\mathbf{C}^{n}$, a theorem of the Paley-Wiener type is obtained to determine the support of a continuous, integrable function from its Radon transform. The construction requires defining an associated function of the Radon transform for each $z \in \mathbf{C}^{n}$. The support of the function is then obtained via analytic multipliers of the associated functions.
\end{abstract}

Given an integrable function $f(x)$, defined on $\mathbf{R}^{n}$, its Radon transform is obtained by integration over hyperplanes $\langle\xi, x\rangle=p$. Here $\xi$ denotes a nonzero vector in $\mathbf{R}^{n}, p$ a real number, and $\langle\cdot, \cdot\rangle$ the usual inner product. The Radon transform is the resulting function of $\xi$ and $p$ given by

$$
\check{f}(\xi, p)=\int_{\mathbf{R}^{n}} f(x) \delta(p-\langle\xi, x\rangle) d x,
$$

where $\delta$ denotes the Dirac delta function. The integral transform defined by (1) has considerable application to the solution of linear partial differential equations. For example, it is rather elementary to show that a homogeneous elliptic partial differential operator is necessarily hypoelliptic (cf. [6]). Other applications of the Radon transform may be found in [4] and [7].

It follows easily from (1), by a change of variables, that the Radon transform of $f\left(x+x_{0}\right)$ is given by

$$
\check{f}\left(\xi, p+\left\langle\xi, x_{0}\right\rangle\right) .
$$

Consequently, if $f(x)$ is continuous then $\check{f}(\xi, p)$ is continuous in $p$. It is not difficult to show that $\breve{f}(\xi, p)$ is, in fact, simultaneously continuous in both $\xi$ and $p$. An analogous result may be established concerning the order of differentiability of $\check{f}(\xi, p)$. Namely, if $f(x)$ is $k$ times continuously differentiable, then $\check{f}(\xi, p)$ is also $k$ times continuously differentiable in $\xi$ and $p$. Proofs of the above statements may be found in [1].

In this paper we consider the Radon transform defined over the complex domain $\mathbf{C}^{n}$. Our primary purpose is obtaining an analog of the classical Paley-Wiener theorem which determines the support of a function. As is the case with the Fourier transform, the support of a function is characterized in terms of Phragmén-Lindelöf indicators; these are associated with its Radon

Presented to the Society, January 28, 1977 under the title Radon transforms over the complex domain; received by the editors December 8, 1977.

AMS (MOS) subject classifications (1970). Primary 44A15.

Key words and phrases. Radon transform, support, analytic multipliers.

C American Mathematical Society 1978 
transform. Specifically, the indicator functions are obtained as analytic multipliers. This is the content of our first two theorems. The remainder of the paper is essentially concerned with presenting some counterexamples. These illustrate the limitations of our results and lead to some interesting questions which seem to be open.

We now list some of the known results concerning the Radon transforms, of functions defined on $\mathbf{C}^{n}$, which will be used in the sequel. Again, the reader is referred to [1] for proofs. By analogy with the real domain $\mathbf{R}^{n}$, the Radon transform is obtained by integrating $f(z)$ over the hyperplane $s=\langle\zeta, z\rangle$ where $\zeta \in \mathbf{C}^{n}(\zeta \neq 0)$ and $s$ is a complex number. The complex inner product is given by

$$
\langle\zeta, z\rangle=\sum_{j=1}^{n} \zeta_{j} \bar{z}_{j}
$$

where $\bar{z}_{j}$ denotes the complex conjugate of $z_{j}$. The Radon transform of $f(z)$ is then given by

$$
\check{f}(\zeta, s)=(i / 2)^{n} \int_{\mathbf{C}^{n}} f(z) \delta(s-\langle\zeta, z\rangle) d z d \bar{z}
$$

where $d z$ and $d \bar{z}$ denote the differential $d z_{1} d z_{2} \cdots d z_{n}$ and its complex conjugate. The appearance of the factor $(i / 2)^{n}$ is explained by computing the Jacobian of the transformation of $z, \bar{z}$ to polar coordinates $r_{1} e^{i \theta_{1}}$, $r_{2} e^{i \theta_{2}}, \ldots, r_{n} e^{i \theta_{n}}$. Since the Jacobian is $(i / 2)^{-n}$, we have merely introduced a scaling factor which ensures that the Radon transform of a real valued function is also real valued. It follows easily from (4) that the Radon transform of $f\left(z+z_{0}\right)$ is given by $\check{f}\left(\zeta, s+\left\langle\zeta, z_{0}\right\rangle\right)$. By analogy with (2) over the real domain this constitutes the first step in establishing that $\check{f}(\zeta, s)$ is continuous provided that $f(z)$ is. More generally, it can be shown that if $f(z)$ is $k$ times continuously differentiable in both $z$ and $\bar{z}$ then $\breve{f}(\zeta, s)$ is $k$ times continuously differentiable with respect to $s$ and $\bar{s}$ (cf. [1]).

Another important property of the Radon transform is that

$$
\check{f}(\alpha \zeta, \alpha s)=\alpha^{-1} \bar{\alpha}^{-1} \check{f}(\zeta, s),
$$

for any complex number $\alpha \neq 0$. We shall make use of (5) in one of two ways. First, by setting $\alpha=1 /\|\zeta\|$, we may view the Radon transform as a function of a unit vector $\zeta$ and the complex number $s$. Secondly, if $\breve{f}(\zeta, s) \neq 0$ for all unit vectors $\zeta$ and some $s$ then this also is valid for $\breve{f}(\zeta,\|s\|)$. We remark that the restriction $\|\zeta\|=1$ leads to an interesting result. If $f(z)$ has bounded support then $f(z)$ vanishes outside $\left\{z \in \mathbf{C}^{n} \mid\|z\|\langle r\}\right.$. Since $|\langle\zeta, z\rangle| \leqslant\|z\|$ for $\|\zeta\|=1$ we conclude that $\check{f}(\zeta, s)=0$ for any $s$ such that $|s|>r$. Thus, if $f(z)$ is continuous with compact support then $f(\zeta, s)$ is also continuous with compact support (cf. [5]). This indicates some relationship between the supports of $f$ and $\check{f}$.

We shall determine a more precise relationship between the support of $f(z)$ 
and the support of $\check{f}(\zeta, s)$. It will be assumed that $f(z)$ is real valued, continuous, and integrable. It is further assumed that $f=f^{+}-f^{-}$has its Radon transform given as $\check{f}^{+}-\check{f}^{-}$. Here $f^{+}$and $f^{-}$denote the Jordan decomposition of $f$. It follows immediately from (4) that $\check{f}^{+}$and $\check{f}^{-}$are also nonnegative. We consider the sets defined as follows:

$$
\begin{aligned}
& S_{f}=\left\{z_{0} \in \mathbf{C}^{n} \mid f\left(z_{0}\right) \neq 0\right\}, \\
& A_{f}=\left\{z_{0} \in \mathbf{C}^{n} \mid \check{f}\left(\zeta,\left\langle\zeta, z_{0}\right\rangle\right) \neq 0 \text { for all } \zeta:\|\zeta\|=1\right\} .
\end{aligned}
$$

By our assumptions, both $S_{f}$ and $A_{f}$ are open. Indeed since $f$ and $\check{f}$ are continuous, the sets where $f$ and $\check{f}$ do not vanish must be open. We shall establish a series of lemmas which indicate the relationship between $S_{f}$ and $A_{f}$. Since $f$ is continuous, the closure of $S_{f}$ is the support of $f$.

LEMMA 1. If $g(z)=f\left(z+z^{\prime}\right)$ then the following statements are valid:

(i) $0 \in S_{g}$ if and only if $z^{\prime} \in S_{f}$,

(ii) $0 \in A_{g}$ if and only if $z^{\prime} \in A_{f}$.

Proof. (i) Evidently, $g(0) \neq 0$ if and only if $f\left(z^{\prime}\right) \neq 0$.

(ii) By virtue of (4),

$$
A_{g}=\left\{z_{0} \in \mathbf{C}^{n} \mid f\left(\zeta,\left\langle\zeta, z_{0}+z^{\prime}\right\rangle\right) \neq 0 \text { for all } \zeta:\|\zeta\|=1\right\} .
$$

Thus, $0 \in A_{g}$ if and only if $z^{\prime} \in A_{f}$.

A simple consequence of Lemma 1 is that 0 is in the convex hull of $S_{g}$ if and only if $z^{\prime}$ is in the convex hull of $S_{f}$. The same is true for the complex convex hull which we now define. Given two points $z$ and $z_{0}$ in $\mathbf{C}^{n}$, the complex line segment between them is defined to be

$$
\left\{\frac{1}{2}(\alpha+1) z+\frac{1}{2}(1-\alpha) z_{0}|| \alpha \mid \leqslant 1\right\} .
$$

The complex convex hull of a set $S$ is, then, the collection of points $z, z_{0}$ in $S$ union the complex line segment defined by (6). In what follows we shall be concerned only with the complex convex hull. This is denoted by $\operatorname{ch}(S)$. Before establishing our results concerning the complex convex hull we need

LEMMA 2. Let $M$ be a nonsingular linear transformation defined on $\mathbf{C}^{n}$, and define $h(z)=g(M z)$. Then the following statements are valid.

(i) $0 \in S_{g}$ if and only if $0 \in S_{h}$,

(ii) $0 \in A_{g}$ if and only if $0 \in A_{h}$.

Proof. (i) Since $M 0=0$ the statement follows immediately.

(ii) The Radon transform of $h(z)$ is given by

$$
\check{h}(\zeta, s)=(i / 2)^{n} \int_{\mathbf{C}^{n}} g(M z) \delta(s-\langle\zeta, z\rangle) d z d \bar{z}=|\operatorname{det}(M)|^{2} g\left(M^{t} \zeta, s\right)
$$

where $M^{t}$ denotes the transpose of $M$, and det denotes the determinant. 
Evidently, $\check{h}(\zeta, 0) \neq 0$ for all $\zeta$ is equivalent to $\check{g}(\zeta, 0) \neq 0$ for all $\zeta$. This proves the lemma.

LEMMA 3. We have the inclusion $A_{f} \subset \operatorname{ch}\left(S_{f}\right)$.

Proof. Let $z_{0}$ be an arbitrary element of $A_{f}$. By Lemma $1,0 \in A_{g}$ where $g(z)=f\left(z+z_{0}\right)$. We show that $0 \in \operatorname{ch}\left(S_{g}\right)$. By the remarks following Lemma 1 , this yields $z_{0} \in \operatorname{ch}\left(S_{f}\right)$.

Suppose, to the contrary, that $0 \in A_{g}$ and $0 \notin \operatorname{ch}\left(S_{g}\right)$. This means that no $z^{\prime}$ and $z$ in $S_{g}$ can satisfy the equation

$$
0=\frac{1}{2}(\alpha+1) z+\frac{1}{2}(1-\alpha) z^{\prime}
$$

for $|\alpha| \leqslant 1$. Equivalently, if $z \in S_{g}$ then $(\alpha+1) /(\alpha-1) z$ is not in $S_{g}$ for any $|\alpha| \leqslant 1$ with $\alpha \neq 1$.

By virtue of Lemma 2, we may assume that the orthogonal unit vectors having 1 in their $j$ th coordinate and 0 elsewhere are each in $S_{g}(j=1$, $2, \ldots, n)$. Indeed, since $S_{g}$ is open we may extract a basis of $n$ vectors. These can be transformed to the unit vectors mentioned above by some matrix $M$.

Since the unit vector $z^{(j)}$ with 1 in its $j$ th coordinate is in $S_{g}$, no vector of the form $(\alpha+1) /(\alpha-1) z^{(j)}$ can be in $S_{g}$. Equivalently, since $(\alpha+1) /(\alpha-$ 1) maps $|\alpha| \leqslant 1$ onto the closed left half plane, no vector in $S_{g}$ can have nonpositive imaginary components. But this means that the hyperplane $z_{1}+z_{2}+\cdots+z_{n}=0$ cannot intersect $S_{g}$. Since $0 \in A_{g}$, no such hyperplane can exist. This contradiction implies that $0 \in \operatorname{ch}\left(S_{g}\right)$, and concludes the proof.

Lemma 4. $S_{f^{+}} \subset A_{f^{+}}$and $S_{f^{-}} \subset A_{f^{-}}$where $f=f^{+}-f^{-}$.

Proof. Since $f^{+}$is nonnegative, the Radon transform $\check{f}^{+}\left(\zeta,\left\langle\zeta, z_{0}\right\rangle\right)>0$ whenever $f^{+}\left(z_{0}\right)>0$. Similarly, the Radon transform of $f^{-}$evaluated at $\zeta,\left\langle\zeta, z_{0}\right\rangle$ is also positive whenever $f^{-}\left(z_{0}\right)>0$. This proves the lemma.

Applying Lemma 3 to $f^{+}$and $f^{-}$we obtain

$$
S_{f^{+}} \subset A_{f^{+}} \subset \operatorname{ch}\left(S_{f^{+}}\right), \quad S_{f^{-}} \subset A_{f^{-}} \subset \operatorname{ch}\left(S_{f^{-}}\right) .
$$

Taking convex hulls of the inclusions in (7) yields

$$
\operatorname{ch}\left(S_{f^{+}}\right)=\operatorname{ch}\left(A_{f^{+}}\right), \quad \operatorname{ch}\left(S_{f^{-}}\right)=\operatorname{ch}\left(A_{f^{-}}\right)
$$

Since

$$
\operatorname{ch}\left(S_{f}\right)=\operatorname{ch}\left(S_{f^{+}} \cup S_{f^{-}}\right) \supset \operatorname{ch}\left(S_{f^{+}}\right) \cup \operatorname{ch}\left(S_{f^{-}}\right)
$$

we have a slightly sharper result than a determination of $\operatorname{ch}\left(S_{f}\right)$. Now for each point $z_{0} \neq 0$ in $\operatorname{ch}\left(A_{f^{+}}\right)$we consider $\check{f}^{+}\left(\zeta,\left\langle\zeta, z_{0}\right\rangle\right)$ evaluated at $\left\langle\zeta, z_{0}\right\rangle=$ $\left\|z_{0}\right\|$. Since $\check{f}^{+}$does not vanish for any $\|\zeta\|=1$, we may set $\zeta=z_{0} /\left\|z_{0}\right\|$ to show that $\check{f}^{+}(\zeta, 1)$ does not vanish for some $\zeta$. By the continuity of the Radon transform, $\check{f}^{+}(\zeta, 1) \neq 0$ in some neighborhood of $z_{0} /\left\|z_{0}\right\|$. From the set of directions $\zeta$ where $\check{f}^{+}(\zeta, 1) \neq 0$ we generate the cone consisting of vectors of the form $\left(\alpha_{1} \zeta_{1}, \alpha_{2} \zeta_{2}, \ldots, \alpha_{n} \zeta_{n}\right)$ for all $\alpha_{j}>0(j=1,2, \ldots, n)$. 
This determines a wedge in $\mathbf{C}^{n}$. Bearing in mind that this construction depends on $z_{0}$, we define the associated function of $f^{+}$at $z_{0}$, to be

$$
F_{z_{0}}^{+}(\alpha \zeta)=\|\zeta\|^{2} \check{f}^{+}(\zeta, 1)
$$

The factor $\|\zeta\|^{2}$ is introduced to compensate for the homogeneity of $\check{f}$; this simplifies the statement of our results. Specifically, the associated function is homogeneous of degree 0 . Since $F_{z_{0}}^{+}$is necessarily bounded on the compact set of vectors $\zeta$ such that $\|\zeta\|=1$, we conclude that $F_{z_{0}}^{+}$is also bounded on $\mathbf{C}^{n}$. This procedure may be carried out verbatim for $z_{0} \in \operatorname{ch}\left(A_{f^{-}}\right)$. As such, we shall phrase our theorems in terms of $f^{+}$only.

THEOREM 1. The convex hull of the support of $f^{+}$, denoted $\operatorname{ch}\left(\operatorname{supp} f^{+}\right)$ coincides with the closure of

$$
\left\{z_{0} \in \operatorname{ch}\left(A_{f^{+}}\right) \backslash\{0\} \mid F_{z_{0}}^{+} \neq 0 \text { on some wedge in } \mathbf{C}^{n}\right\} .
$$

Proof. As noted above, each $z_{0} \neq 0$ in $\operatorname{ch}\left(A_{f^{+}}\right)$induces an associated function which is nonzero on some wedge. By Lemma $2, z_{0} \in \operatorname{ch}\left(S_{f^{+}}\right)$. This establishes that the set defined by (8) is contained in $\operatorname{ch}\left(S_{f^{+}}\right)$. The inclusion remains valid for closures of these sets.

Conversely, suppose $z_{0}$ is in $\operatorname{ch}\left(\operatorname{supp} f^{+}\right)$. Then there exists a sequence $\left\{z_{k}\right\}$, with each $z_{k} \neq 0$, contained in $\operatorname{ch}\left(S_{f^{+}}\right)$which converges to $z_{0}$. By Lemma 3, each $z_{k}$ is in $\operatorname{ch}\left(A_{f^{+}}\right)$. Since $F_{z_{0}}^{+} \neq 0$ for each $z_{k}$, we have shown that each point $z_{0}$ in $\operatorname{ch}\left(\operatorname{supp} f^{+}\right)$is a limit of a sequence in (8). This means that $\operatorname{ch}\left(\operatorname{supp} f^{+}\right)$is contained in the closure of the set defined in (8)?

In view of Theorem 1 , we consider entire analytic functions as multipliers of each $F_{z_{0}}^{+}$. If $G(\zeta)$ is such an entire function then the product $F_{z_{0}}^{+}(\zeta) G(\zeta)$ is bounded provided $G(\zeta)$ is bounded on the wedge where $F_{z_{0}}^{+} \neq 0$. Conversely, if $G(\zeta)$ is unbounded on any wedge whose closure is contained in the wedge where $F_{z_{0}}^{+} \neq 0$, then the product is unbounded. An example of an unbounded multiplier is

$$
G(\zeta)=\exp \sum_{j=1}^{n}\left(-\lambda_{j} \zeta_{j}\right)^{2 k_{j}}
$$

where each $\lambda_{j}$ is a complex number of modulus 1 and $k_{1}, \ldots, k_{n}$ are positive integers. This function is bounded on a wedge of aperture $\pi / 2 k_{j}$ centered at $\arg \lambda_{j}$, in its respective coordinates. If the $\lambda_{j}$ 's and $k_{j}$ 's are properly chosen, (9) provides an unbounded multiplier for any $F_{z_{0}}^{+}$. Specifically, we set $\lambda_{j}$ equal to the $j$ th component of $z_{0} /\left\|z_{0}\right\|$ and choose the $k_{j}$ 's sufficiently large.

THEOREM 2. The convex hull of the support of $f^{+}$coincides with the closure of

$$
\left\{z_{0} \in \operatorname{ch}\left(A_{f^{+}}\right) \backslash\{0\} \mid F_{z_{0}}^{+} \text {has unbounded analytic multipliers }\right\} \text {. }
$$

Proof. By the previous discussion, $\operatorname{ch}\left(\operatorname{supp} f^{+}\right)$is contained in the closure of the set defined by (10). Conversely, the set defined in (10) is evidently a 
subset of $\operatorname{ch}\left(A_{f^{+}}\right) \backslash\{0\}$. This, in turn, is a subset of $\operatorname{ch}\left(S_{f^{+}}\right)$. Taking closures of these sets completes the proof.

The functions defined by (9), or any analytic function which is unbounded on some wedge, become indicator functions for the support of $f^{+}(z)$. Indeed, this is the content of Theorem 2. The results are identical for $f^{-}(z)$. It is interesting to note the relation between Theorem 2 and the Phragmén-Lindelöf theorem on a wedge (cf. [3]). This relation is similar to that between the Paley-Wiener theorem for Fourier transforms and the Phragmén-Lindelöf theorem for a strip.

There are two directions in which we might attempt to generalize our results. These are removing the assumptions of continuity or integrability. The integrability of $f(z)$ ensures the existence of the Radon transform. Indeed, there exist functions in $L^{p}\left(\mathbf{C}^{n}\right)$ which are not integrable over any $n-1$ dimensional complex hyperplane. This follows immediately from the equivalent statement concerning $2 n-2$ dimensional real hyperplanes of $\mathbf{R}^{2 n}$ (cf. [8]). The continuity of $f$ also is essential for the validity of Theorem 2 . To show this we construct a nonnegative, integrable function $f(z)$ for which $F_{z_{0}}(\zeta)$ has zeroes on every wedge in $\mathbf{C}^{n}$. The example is defined on $\mathbf{C}^{2}$ by setting $f\left(z+z_{0}\right)=g(z)$ where $g(z)$ is defined as follows. Let $g\left(z_{1}, z_{2}\right)=g\left(x_{1}\right.$ $\left.+i y_{1}, x_{2}+i y_{2}\right)$ equal 0 provided $n_{1} x_{1}+n_{2} y_{1}+n_{3} x_{2}=0$ and $n_{2} x_{1}-n_{1} y_{1}$ $-n_{3} y_{2}=0$ for some integers $n_{1}, n_{2}, n_{3}$ and $x_{1}^{2}+y_{1}^{2}+x_{2}^{2}+y_{2}^{2}>1$. If any of these conditions is violated we let $g=1$. The Radon transforms of $f$ and $g$ are related by $\check{f}\left(\zeta,\left\langle\zeta, z_{0}\right\rangle\right)=\check{g}(\zeta, 0)$. By the homogeneity, the vanishing of $\check{g}(\zeta, 0)$ is invariant under division by one of the components of $\zeta$. At least one of $\zeta_{1}, \zeta_{2}$ must be nonzero, say $\zeta_{2}$. Then consider $\check{g}$ evaluated at the vector with components $\zeta_{1} / \zeta_{2}, 1$. It is easily shown that $\check{g}\left(\left(\zeta_{1} / \zeta_{2}, 1\right), 0\right)$ vanishes if and only if

$$
\zeta_{1} / \zeta_{2}=n_{1} / n_{3}+n_{2} / n_{3} \text {. }
$$

Since any Gaussian rational $a+i b$ with $a, b$ rational can be written in the form of (11), we conclude that $\check{g}$ vanishes on what are termed rational directions $\zeta$. Since the set of rational directions is dense in the set of all direction vectors, we conclude that $\check{g}(\zeta, 0)$ vanishes on every open wedge in $\mathbf{C}^{n}$. Consequently, $\check{f}\left(\zeta,\left\langle\zeta, z_{0}\right\rangle\right)$ has an associated function $F_{z_{0}}(\zeta)$ which vanishes on a dense subset of directions.

Since continuity plays such an important role in our theorems it is of interest to determine when $f(z)$ is continuous based upon the behavior of $\check{f}(\zeta, s)$. One result is as follows:

The function $f(z)$ is continuous at $z_{0}$ if $\check{f}\left(\zeta,\left\langle\zeta, z_{0}\right\rangle\right)$ is $n-1$ times continuously differentiable with respect to both $s$ and $\bar{s}$. The proof of the statement follows immediately from the inversion formula in [1]. Since the continuity of $f(z)$ does not imply the differentiability of $\breve{f}(\zeta, s)$ this result is somewhat unsatisfactory. It would be of interest to obtain precise results relating the smoothness of $f$ to that of $\check{f}$. The identical situation and problem 
exists for the Radon transform on $\mathbf{R}^{n}$. Here $\check{f}(\xi, p)$ must be $n-1$ times continuously differentiable to imply the continuity of $f(x)$.

We conclude by mentioning an example, on the real domain, which provides a partial answer to the above problem. Let $f(x)$ be the characteristic function of the unit ball in $\mathbf{R}^{2 l+1}$. It is not difficult to show that

$$
\check{f}(\xi, p)=\left\{\begin{array}{l}
\frac{\pi^{l}}{l !}\|\xi\|^{-1}\left(1-p^{2} /\|\xi\|^{2}\right)^{l} \text { for }|p| \leqslant\|\xi\|, \\
0 \text { for }|p|>\|\xi\| .
\end{array}\right.
$$

A computation shows that $\check{f}(\xi, p)$ is $l-1$ times continuously differentiable. Since $f(x)$ is not continuous, we have shown the following.

THEOREM 3. On $\mathbf{R}^{2 l+1}$ the continuous differentiability of order $l-1$ of $\check{f}$ is not sufficient to imply the continuity of $f$.

Question. Does $\check{f}(\xi, p)$ being $l$ times continuously differentiable with respect to $p$ imply that $f(x)$ is continuous?

We remark that a similar example, yielding similar results, may be obtained over $\mathbf{C}^{n}$. Again, it remains to be seen to what extent these results may be sharpened.

\section{REFERENCES}

1. I. M. Gel'fand, M. I. Graev and N. Ya Vilenkin, Integral geometry and representation theory, Academic Press, New York, 1966.

2. I. M. Gel'fand and G. E. Shilov, Properties and operations, Academic Press, New York, 1966.

3. , Functions and generalized spaces, Academic Press, New York, 1966.

4. F. John, Plane waves and spherical harmonics, Interscience, New York, 1955.

5. J. V. Peters, Radon transforms over the real and complex domain, Doctoral Thesis, Stevens Institute of Technology, Hoboken, N. J., 1976.

6. G. E. Shilov, Generalized functions of partial differential equations, Gordon and Breach, New York, 1968.

7. K. T. Smith, D. C. Solmon and S. L. Wagner, Practical and mathematical aspects of the problem of reconstructing objects from radiographs. Bull. Amer. Math. Soc. 83 (1977), 1227-1270.

8. D. C. Solmon, A note on K-plane integral transforms, Math. Anal. Appl. (to appear).

Department of Mathematics, St. Bonaventure University, St. Bonaventure, New York 14778

Current address: 88-08 235th Street, Queens Village, New York 11427 